\title{
Human Umbilical Cord Mesenchymal Stem Cells in the Treatment of Secondary Progressive Multiple Sclerosis
}

\author{
Lu $Z^{1,2}$, Zhao $H^{1,2}, X u J^{3}$, Zhang $Z^{1}$, Zhang $X^{1}$, Zhang $Y^{1}$, Liu $Z^{1,2}$ and $X u Y^{1,2 *}$
}

${ }^{1}$ Department of Neurology, Drum Tower Hospital, Nanjing Medical University, Nanjing, Jiangsu, PR China

${ }^{2}$ Department of Neurology, Affiliated Drum Tower Hospital, Nanjing University Medical School, Nanjin, Jiangsu, PR China

${ }^{3}$ Department of Neurology, Brain Hospital, Nanjing, Jiangsu, PR China

\begin{abstract}
Background: Multiple sclerosis (MS) is an irreversible and demyelinating disease of the brain and spinal cord that causes significant disability in young people. However, therapeutic options to effectively stop the pathological progression of MS are limited. This study aimed to evaluate the safety and efficacy of human umbilical cord-derived mesenchymal stem cells (hUC-MSCs) in the treatment of secondary progressive multiple sclerosis.
\end{abstract}

Methods: Eight patients with secondary progressive multiple scleroses were recruited from May 2010 and December 2010. Participants received intrathecal intravenous injections of hUC-MSCs. All patients were evaluated by Expanded Disability Status Scale, frequency of relapse, lymphocyte classification, magnetic resonance imaging (MRI) characteristics and adverse events from before treatment to up to 18 months after the infusion.

Results: The study suggested that, among the eight cases, six showed therapeutic improvement after hUCMSCs treatment and in all eight patients, relapse frequencies decreased to $36.4 \%$ of those before the treatment $(1.2$ \pm 0.5 vs. $3.3 \pm 0.7, p<0.05$ ). Flow cytometry assay (FACS) indicated that blood T cells and B cells were inhibited after treatment. MRI characteristics also showed decreased volume and severity of lesions, with few adverse events.

Conclusion: hUC-MSCs can alleviate disability and reduce relapse of secondary progressive multiple sclerosis by modulating immune responses, which also had been proved to be safe in MS patients. These findings suggest that transplantation of hUC-MSCs may be a potential therapy for MS.

Keywords: Human umbilical cord-derived mesenchymal stem cells; Progressive multiple sclerosis; Safety; Therapy

\section{Introduction}

Multiple sclerosis (MS) affects over 1.3 million people worldwide and is the most common cause of disability in young adults [1]. MS is an inflammatory disease affecting the central nervous system (CNS). Pathological characteristics include inflammatory demyelination, axonal injury and partial remyelination [2]. Relapsing Remitting MS (RRMS), the most common type of MS, exhibits full recovery between attacks at an early stage of disease, but more than 50\% of RRMS cases eventually enter a chronic progressive phase (secondary progressive MS (SPMS)) with accumulating neurological dysfunction [3]. The number of RRMS treatment options, such as immunomodulatory and immunosuppressive therapies, is constantly growing, but treatment options for patients suffering from progressive forms of multiple sclerosis (MS) remain inadequate [4].

Stem cell therapy, especially the transplantation of mesenchymal stem cells (MSCs), has emerged as a potential treatment for MS [5]. MSCs are commonly isolated from bone marrow (BM), adipose tissue, umbilical cord blood, placenta, embryo, and thymus. MSCs, as multipotent cells, possess immunomodulatory properties that can be explored for non-autologous (allogeneic) stem cell therapy to modulate aberrant immune responses. They also synthesize and secrete multiple growth factors/cytokines (trophic factors) [6]. Clinical trials using human bone marrow mesenchymal stem cells (BM-MSC) have been initiated for immune disorders and preclinical data have demonstrated BM-MSC transplantation alleviated the symptoms of experimental autoimmune encephalomyelitis (EAE) [7].

Given the fact that harvesting BM-MSCs is painful, invasive and low yield, alternative sources of MSCs may be more useful. Therefore,
MSCs derived from human umbilical cord blood (hUC) have attracted attention [8]. Superior to BM-MSCs, human umbilical cord blood mesenchymal stem cells (hUC-MSCs) have significantly lower human leukocyte antigen-1 (HLA-1) expression, greater expansion capability and faster growth in vitro [9].

In this study, eight patients were enrolled with the aim to evaluate the clinical efficacy and safety of hUC-MSCs for patients with progressive MS in a Chinese population.

\section{Methods and Materials}

\section{Patient enrollment}

Between May 2010 and December 2010, eight MS patients were enrolled in this study, six recruited from the Department of Neurology of the Affiliated Drum Tower Hospital of Nanjing University Medical School and two recruited from the Department of Neurology of Nanjing Brain Hospital. The patients aged 18 to 59 years. This study was approved by the ethics committees of the Affiliated Drum Tower

*Corresponding author: Yun Xu, MD, PhD, Department of Neurology, Affiliated Drum Tower Hospital, Nanjing University Medical School, 321 Zhongshan Road, Nanjing, Jiangsu, 210008, P. R. China, Tel: +86 25 83105208; Fax: +86 25 83317016; E-mail: xuyun20042001@aliyun.com

Received October 25, 2013; Accepted November 25, 2013; Published November 27, 2013

Citation: Lu Z, Zhao H, Xu J, Zhang Z, Zhang X, et al. (2013) Human Umbilical Cord Mesenchymal Stem Cells in the Treatment of Secondary Progressive Multiple Sclerosis. J Stem Cell Res Ther S6: 002. doi:10.4172/2157-7633.S6-002

Copyright: (C) 2013 Lu Z, et al. This is an open-access article distributed under the terms of the Creative Commons Attribution License, which permits unrestricted use, distribution, and reproduction in any medium, provided the original author and source are credited. 
Hospital of Nanjing University Medical School and Nanjing Brain Hospital (clinical trials government identifier: NCT01364246). All patients provided written informed consent.

\section{Enrollment criteria}

The following enrollment criteria were used:

1) Diagnosed as clinical SPMS according to McDonald criteria by experienced neurologists $[10,11]$;

2) Aged 18 to 65 years;

3) Duration of disease longer than 4 years EDSS score 3.0 to 8.0 [12];

4) Failure to respond to the currently available and registered agents for MS, as manifested by an EDSS score increase of at least 1 degree or the occurrence of at least 2 major relapses during the previous year. Relapse was defined by the revised McDonald criteria [13].

\section{Exclusion criteria}

The following exclusion criteria were used:

1) Other systemic active disease;

2) Participation in other experimental protocols during the 3 months before the trial;

3) Autoimmune condition unrelated to MS or allergies;

4) Pregnant or possibly pregnant;

5) Inability to fully understand the treatment protocol or unable to sign informed consent;

6) Presence of $\operatorname{HIV}(+)$, Tumor marker $(+)$, brain tumor or $\mathrm{BP} \geq$ $200 \mathrm{mmHg} / 110 \mathrm{mmHg}$;

7) Impracticality, as determined by clinician, of at least 12 month follow-up.

\section{MRI analysis}

All patients underwent cerebral and spinal cord MRI. The patients were scanned on a 1.5 T MRI (Achieva, Philips) including T1-weighted spin-echo (SE) axial slices, with and without gadolinium, as well as T2weighted SE axial slices. All patients have T2-intense periventricular MRI abnormalities and two or more lesions. MRI diagnosis followed Barkhof criteria [13].

\section{Preparation of hUC-MSCs}

hUC-MSCs were from the Jiangsu Provincial Stem Cell Engineering Research Center (Beike Bio-Technology). Fresh umbilical cords (UCs) were obtained from healthy puerper as in a local maternity hospital after normal deliveries, under approved clinical protocol including informed consent donor forms, then processed as quickly as possible. First, the UCs were rinsed twice in phosphate buffered saline (PBS) with antibiotics, then cut into 1-2 mm pieces and floated in Dulbecco's modified Eagle's medium (DMEM)/F12 and medium (DF12) containing human platelet-derived growth factor-BB (PDGF-BB), transforming growth factor (TGF)- $\beta 1$, basic fibroblast growth factor (bFGF) and epidermal growth factor (EGF). They were then incubated at $37^{\circ} \mathrm{C}$ in a humidified atmosphere consisting of $5 \% \mathrm{CO}_{2}$ for about 10 days. The resulting well-developed colonies of fibroblast-like cells were then ready for further expansion.

\section{hUC-MSCs transplantation}

All infused hUC-MSCs were dealed with rigorous purification and quality control. The Mesenchymal stem cells we used were the third generation and the solution was $0.9 \%$ sodium chloride. The first day of transplantation was defined as "Day 0", subsequent days was "Day 1" and so on. On Day 0, the patients received intravenous injection with $40 \mathrm{ml}\left(2 \times 10^{7}\right)$ cells, followed with an intrathecal injection of $1 \mathrm{ml}(2$ $\times 10^{7}$ ) hUC-MSCs. Then on Day 7, Day 14, and Day 21, the patients received an intravenous injection of $20 \mathrm{ml}\left(2 \times 10^{7}\right)$ cells. The immuneenhancing or immunosuppressive medicine was not allowed during hUC-MSCs transplant regimen.

\section{Evaluation of efficacy on patients}

Efficacy was evaluated using the following factors:

1) Expanded Disability Status Scale (EDSS) evaluate the disability status of patients before and after treatment.

2) Relapse rate after treatment. Relapse was defined by the revised McDonald criteria [14].

3) Changes in magnetic resonance imaging (MRI).

The evaluation was completed by a neurologist who was not familiar with the treatment process.

\section{Evaluation of safety on patients}

Safety was assessed throughout the study via physical examinations, vital signs, clinical laboratory evaluations and monitoring of immediate or delayed adverse events (AEs) such as allergic reactions (tachycardia, fever), respiratory failure, local complications (dizziness, lumbago, headache and local or intracranial infection related to lumbar puncture), and tumor formation. The following assessments were performed:

1) Blood tests, including blood routine, blood lipids, blood glucose and electrolytes, liver and kidney function, coagulation function, blood antibodies of autoimmune diseases;

2) Tumor markers determined by direct chemiluminescent immunoassay, including AFP, CEA, NSE, CY211, CA199, CA125, CA153, CA50, CA242, CA724; HIV, HBV, HCV;

3) CMV and syphilis measured by ELISA;

4) EBV determined by PCR;

5) Urine and stool routine; and

6) Electrocardiography, echocardiography, chest X-ray, abdominal color Doppler ultrasound examination and electroencephalogram.

All examinations were completed before and after transplantation and at months 3, 6, 12 and 18 after therapy.

\section{Flow Cytometry Assay (FACS)}

For evaluating the lymphocyte classification before and after hUC-MSC transplantation, $3 \mathrm{ml}$ peripheral blood was collected from each patient with ethylenediamine tetraacetic acid (EDTA) as the anticoagulant and using two panels of labeled monoclonal antibodies (biosciences): CD4-FITC/CD29-PE/CD3-PerCP and CD3-APC/CD4FITC/CD8-PE. Then the stained cells were assessed using a fluorescenceactivated cell sorter Calibur flow cytometer (BD Biosciences). Data analysis was performed using CellQuest Pro software (BD Biosciences). 
Citation: Lu Z, Zhao H, Xu J, Zhang Z, Zhang X, et al. (2013) Human Umbilical Cord Mesenchymal Stem Cells in the Treatment of Secondary Progressive Multiple Sclerosis. J Stem Cell Res Ther S6: 002. doi:10.4172/2157-7633.S6-002

Page 3 of 7

\section{Statistical analysis}

Statistical analyses were performed using SPSS 17.0 software (Bizinsight, Shanghai, China). Data were presented as mean \pm SD. One-way analysis of variance (ANOVA) was used for comparison of intergroup differences. Post-hoc Newman-Keuls testing was used for multiple groups. The $\chi^{2}$ test was used for analyzing data frequency. A probability of $\mathrm{p}<0.05$ was considered to be significant.

\section{Results}

\section{Properties of hUC-MSCs in vitro}

The viability of hUC-MSCs was $92 \%$ (Figure $1 \mathrm{~A}$ ). Flow cytometry assay showed that the cells were negative with CD34, CD45, CD14, CD79, HLA-DR and positive with CD105, CD73 and CD90 (Figure 1B). Meanwhile, bacterial and hepatic viruses and HIV were tested negative to ensure treatment safety (Table 1).

\section{Efficacy and safety of hUC-MSC therapy in patients with MS}

The baseline characteristics of eight MS patients are described in Table 2. The average improvement ratios of EDSS levels were $11.2 \%$ $\pm 8.5 \%, 10.4 \% \pm 13.9 \%, 8.1 \% \pm 17.0 \%, 12.5 \% \pm 18.4 \%, 13.7 \% \pm 15.5 \%$, $9.8 \% \pm 20.6 \%$ and $10.1 \% \pm 18.1 \%$, respectively, at months $1,3,6,9,12$, 15 and 18 after treatment (Figure 2 and Table 3). Assessment at the 18th month revealed EDSS improvement in 6/8, stabilization in $1 / 8$, and stabilization after worsening in $1 / 8$ patients. The relapses rate was reduced in $7 / 8$, stabilization in $1 / 8$ and relapses in all patients decreased to $36.4 \%$ after treatment at 18 months $((1.2 \pm 0.5$ vs. $3.3 \pm 0.7, \mathrm{p}<0.05)$ (Figure 3 and Table 4). No adverse events occurred from due to the hUC-MSC transplant procedure as of 18 months after treatment.

\section{Lymphocytes change in blood}

Immunological analysis indicated a decrease in the proportion of

\begin{tabular}{|c|c|c|c|}
\hline $\begin{array}{c}\text { Surface } \\
\text { markers }\end{array}$ & $\begin{array}{c}\text { Results } \\
\text { (positive rate) }\end{array}$ & Microbiological and other detection & Results \\
\hline CD73 & $96.5 \%$ & Aerobic bacteria & Negative \\
\hline CD90 & $98.4 \%$ & Anaerobic bacteria & Negative \\
\hline CD105 & $97.0 \%$ & Fungus & Negative \\
\hline CD14 & $0.4 \%$ & HBsAg & Negative \\
\hline CD34 & $0.2 \%$ & HBcAb & Negative \\
\hline CD45 & $0.2 \%$ & HCV-Ab & Negative \\
\hline CD79 & $0.5 \%$ & Anti-HIV(I+II) & Negative \\
\hline HLA-DR & $0.4 \%$ & CMV-lgM & Negative \\
\hline- & - & HTLV-I/II & Negative \\
\hline- & - & Anti-EBV antibody & Negative \\
\hline- & - & Syphilis serology test & Negative \\
\hline- & - & Endotoxin & $2.0 \mathrm{EU}$ \\
\hline- & - & ALT & $10 \mathrm{U} / \mathrm{L}$ \\
\hline- & & amount of residue (growth factor) & $0.1 \mathrm{ng} / \mathrm{ml}$ \\
\hline
\end{tabular}

Abbreviations: HLA-DR: Human Leukocyte Antigen DR; HBsAg, Hepatitis B Surface Antigen; HBcAb: Hepatitis B Core Antibody; HCV-Ab: Hepatitis C Virus Antibody; Anti-HIV(I+II): Human Immunodeficiency Virus Antibodies I and II; CMVIgM: Cytomegalovirus IgM; HTLV-I/II: Human T Lymphocytic Leukemia Virus; AntiEBV Antibody: Epstein-barr Virus Antibodies; ALT: Aspartate Aminotransferase.

Table 1: Properties and safety tests of hUC-MSCs.

$\mathrm{T}$ and $\mathrm{B}$ cells in $7 \mathrm{MS}$ patients. Two had a decrease in the proportion of NK cells while others had an increase (Figure 4).

\section{MRI characteristics}

Brain and spinal MRIs were performed before and after hUCMSC treatment. MS was characterized by diffuse or multifocal intramedullary lesions involving the periventricular or spinal cord, with or without enhancement. The number and scope of T1-weighted lesion and T2-weighted lesion volumes were smaller twelve months after
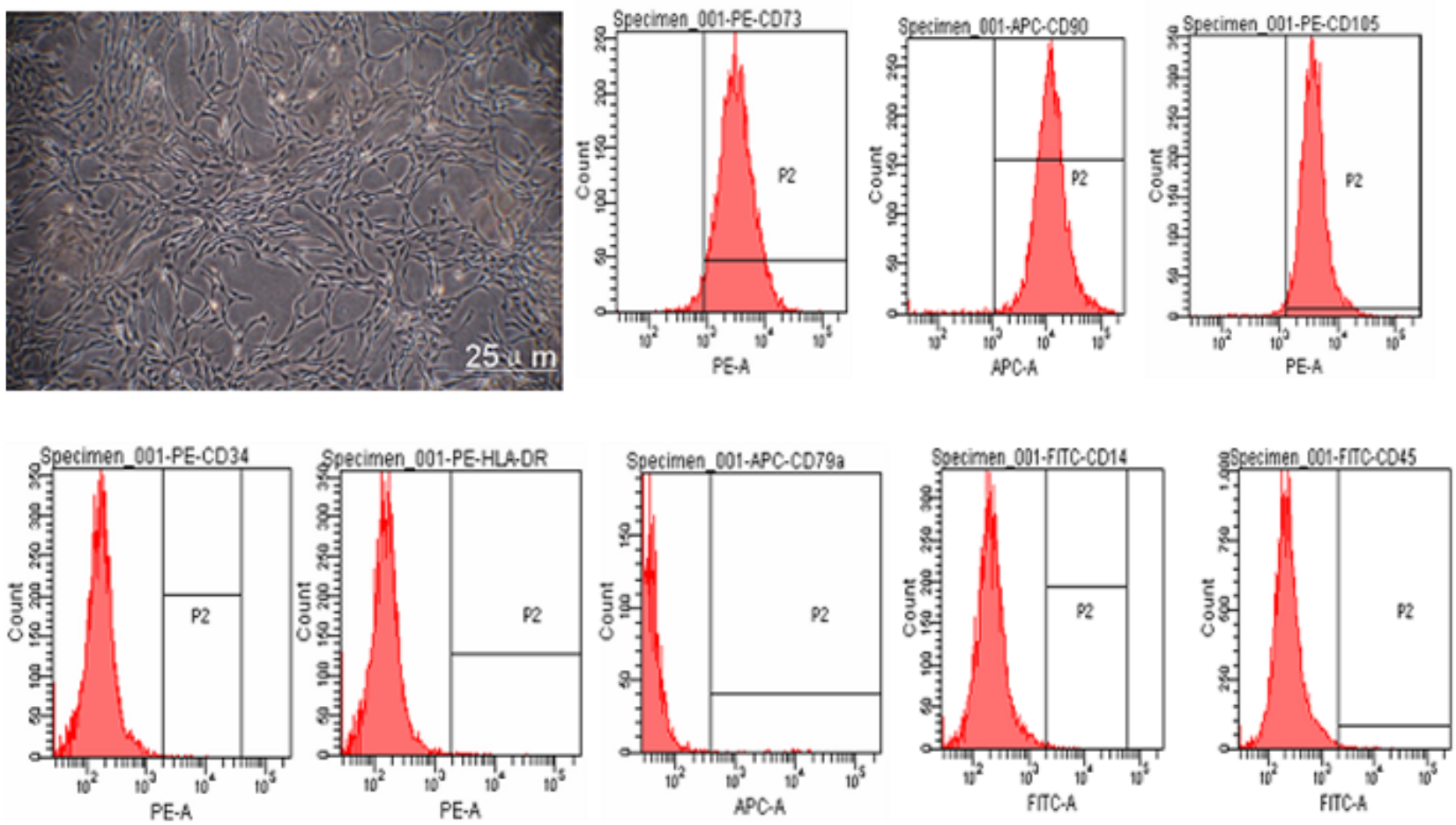

Figure 1: The picture and surface markers of human umbilical mesenchymal stem cells (hUC-MSCs). (A) In vitro, hUC-MSCs were fibroblast-like, and the viability was $92 \%$. (B) The hUC-MSCs were positive for CD105, CD73, CD90 and negative for CD34, CD45, CD14, CD79, HLA-DR according to flow cytometric analysis. 
Citation: Lu Z, Zhao H, Xu J, Zhang Z, Zhang X, et al. (2013) Human Umbilical Cord Mesenchymal Stem Cells in the Treatment of Secondary Progressive Multiple Sclerosis. J Stem Cell Res Ther S6: 002. doi:10.4172/2157-7633.S6-002

Page 4 of 7

\begin{tabular}{|c|c|c|c|c|c|c|c|c|}
\hline \multirow{2}{*}{ Variable } & \multicolumn{8}{|c|}{ Patient No. } \\
\hline & Case 1 & Case 2 & Case 3 & Case 4 & Case 5 & Case 6 & Case 7 & Case 8 \\
\hline Sex & $\mathrm{F}$ & $\mathrm{F}$ & $\mathrm{F}$ & $\mathrm{F}$ & $\mathrm{F}$ & M & $\mathrm{F}$ & M \\
\hline Age at onset, y & 52 & 52 & 26 & 25 & 22 & 14 & 49 & 36 \\
\hline Age at study enrollment, y & 59 & 57 & 41 & 30 & 26 & 18 & 54 & 40 \\
\hline Relapses since disease onset, no. & 21 & 3 & 5 & 12 & 3 & 3 & 3 & 2 \\
\hline Visual evoked potential & Abnormal & Abnormal & Abnormal & Abnormal & Abnormal & Abnormal & Abnormal & Abnormal \\
\hline Previous therapy & CS, IVIG & CS, IVIG & $\mathrm{CS}, \mathrm{IFN}, \mathrm{OA}$ & CS, IFN & CS & CS, IFN & CS, IVIG & CS, IVIG \\
\hline
\end{tabular}

Abbreviations: CS: corticosteroids; IVIG: Intravenous Immunoglobulin; IFN: Interferon; ON: Optic Neuritis; TM: Transverse Myelitis; OA: Oral Azathioprine.

Table 2: Characteristics of MS patients.

Fig 2(A)

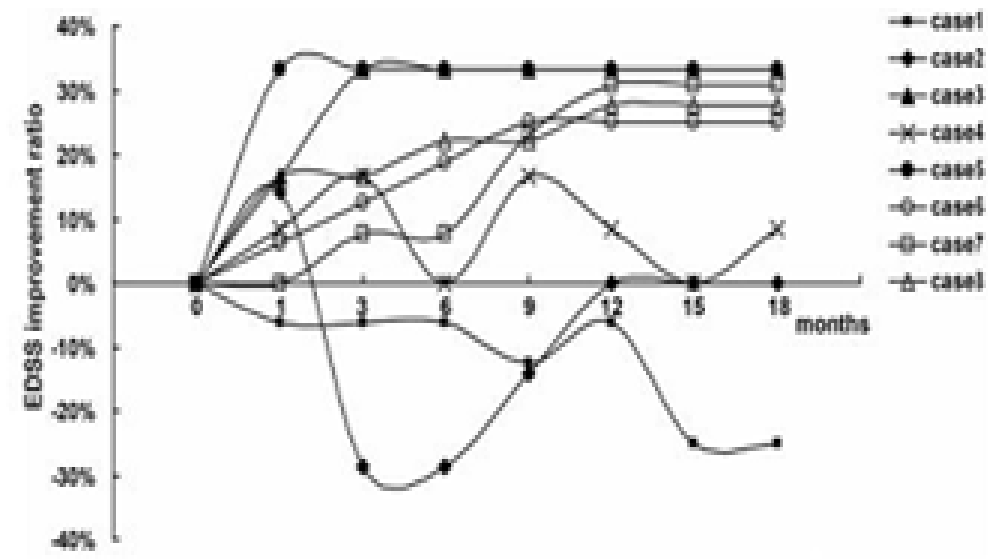

2(B)

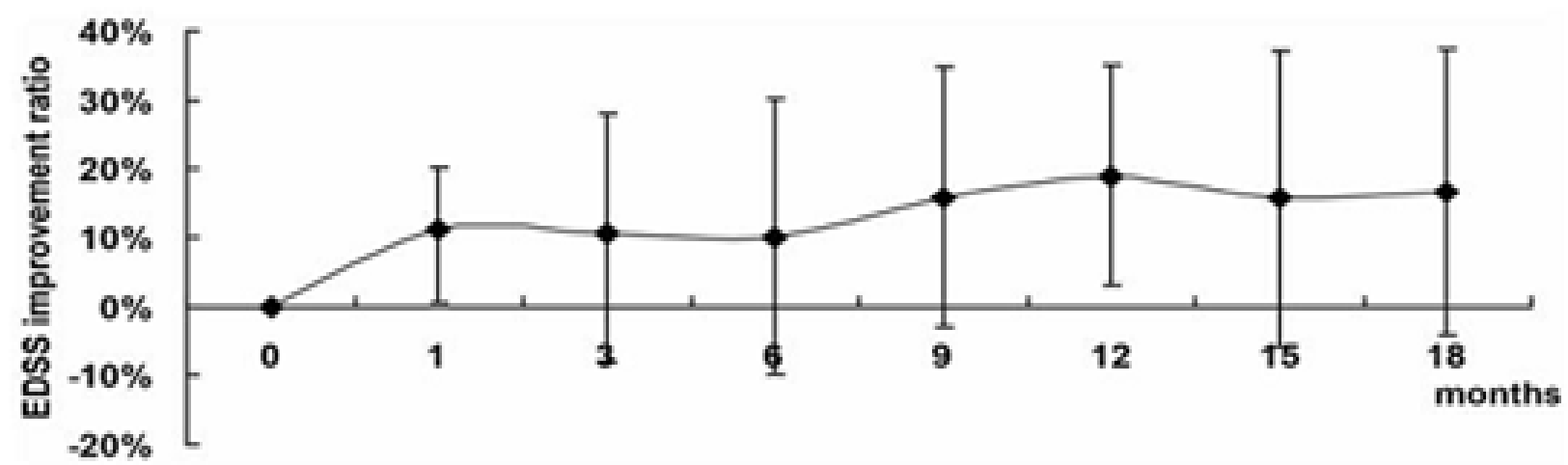

Figure 2: The improvement ratio of EDSS levels during the study. (A) Assessment at month 18 revealed patients (case 3, case 4,case 5, case 6 , case 7 , case 8 ) improved, 1 patient (case 2) stabilized, 1 patient (case 1) got worse. (B) The average improvement ratio of EDSS levels were $11.2 \% \pm 8.5 \%, 10.4 \% \pm 13.9 \%, 8.1 \% \pm$ $17.0 \%, 12.5 \% \pm 18.4 \%, 13.7 \% \pm 15.5 \%, 9.8 \% \pm 20.6 \%$ and $10.1 \% \pm 18.1 \%$, respectively, at month $1,3,6,9,12,15$ and 18 after treatment.

\begin{tabular}{|c|c|c|c|c|c|c|c|c|}
\hline \multicolumn{9}{|c|}{ Score at the follow-up month } \\
\hline Patient No & O(Baseline) & 1 & 3 & 6 & 9 & 12 & 15 & 18 \\
\hline Case 1 & 8 & 8.5 & 8.5 & 8.5 & 9 & 8.5 & 10 & 10 \\
\hline Case 2 & 3.5 & 3 & 4.5 & 4.5 & 4 & 3.5 & 3.5 & 3.5 \\
\hline Case 3 & 3 & 2.5 & 2 & 2 & 2 & 2 & 2 & 2 \\
\hline Case 4 & 6 & 5.5 & 5 & 6 & 5 & 5.5 & 6 & 5.5 \\
\hline Case 5 & 3 & 2 & 2 & 2 & 2 & 2 & 2 & 2 \\
\hline Case 6 & 8 & 7.5 & 7 & 6.5 & 6 & 6 & 6 & 6 \\
\hline Case 7 & 6.5 & 6.5 & 6 & 6 & 5 & 4.5 & 4.5 & 4.5 \\
\hline Case 8 & 9 & 7.5 & 7.5 & 7 & 7 & 6.5 & 6.5 & 6.5 \\
\hline
\end{tabular}

Table 3: Expanded disability status scale scores during the study. 


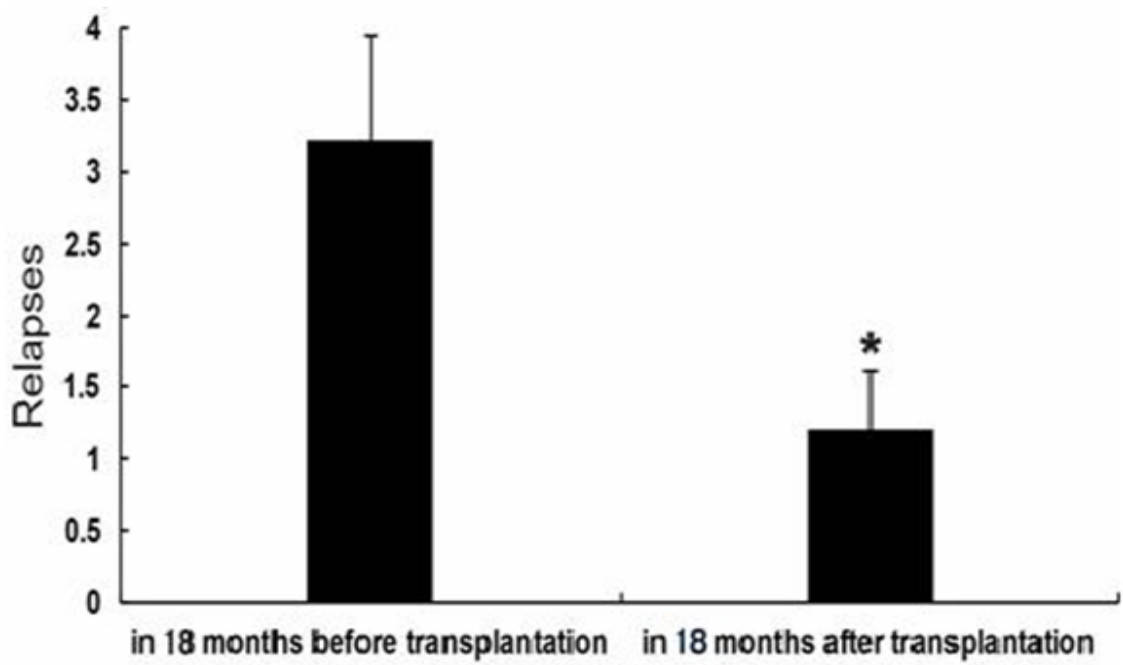

Figure 3: Relapses of patients during the study. The average relapses in all eight patients at 18 months after transplantation decreased significantly compared with that before transplantation: $3.3 \pm 0.7$ vs. $1.2 \pm 0.5 .{ }^{*} P<0.05$.

\begin{tabular}{|c|c|c|}
\hline \multirow[b]{2}{*}{ Patient No. } & \multicolumn{2}{|c|}{ The relapses rate } \\
\hline & 18 months before transplantation & 18 months after transplantation \\
\hline Case 1 & 7 & 4 \\
\hline Case 2 & 2 & 2 \\
\hline Case 3 & 2 & 0 \\
\hline Case 4 & 6 & 3 \\
\hline Case 5 & 2 & 0 \\
\hline Case 6 & 3 & 0 \\
\hline Case 7 & 2 & 0 \\
\hline Case 8 & 1 & 0 \\
\hline
\end{tabular}

Table 4: The relapses rate 18 months before and after transplantation.

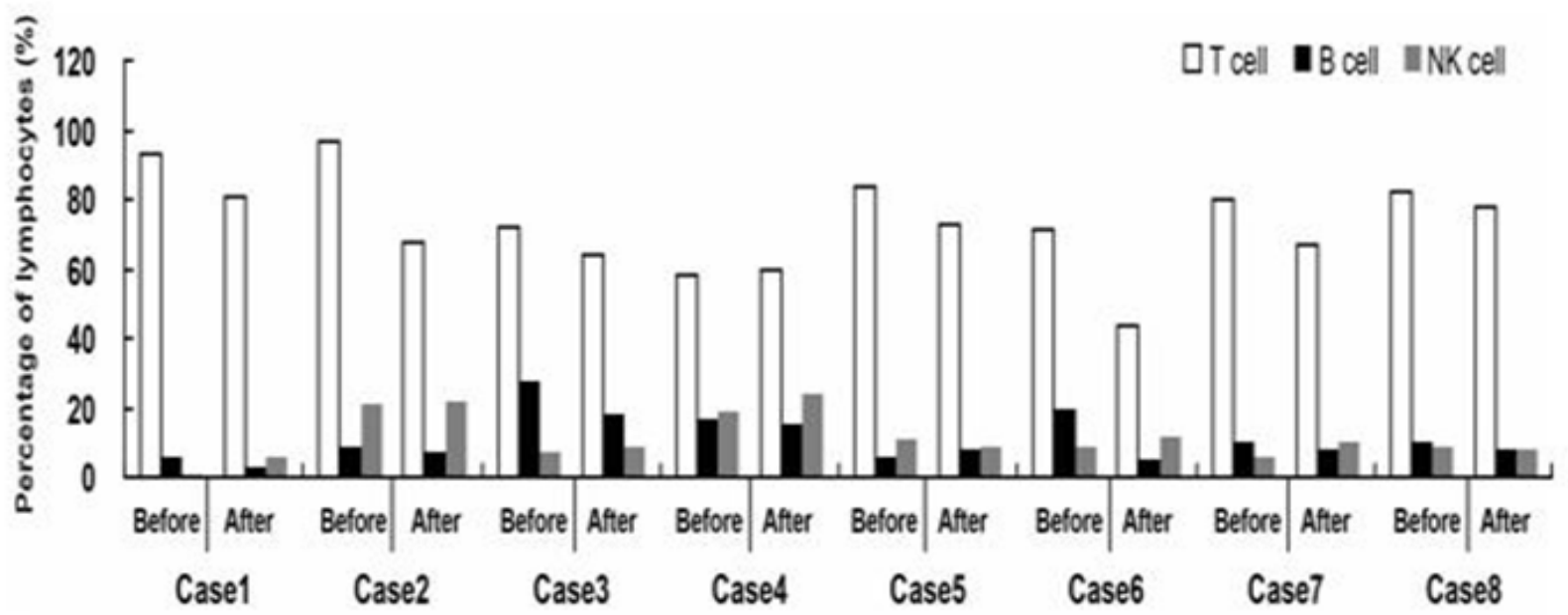

Figure 4: Lymphocyte classification. Lymphocyte classification revealed a decrease in the proportion of T cell and B cells in seven MS patients. Two of the eight patients had a decrease in the proportion of NK cells, while others had an increase.

transplantation. The gadolinium (Gd)-enhancement were decreased in two patients compared to the baseline MRI scan (Figure 5).

\section{Discussion}

This study first reported that human umbilical cord mesenchymal stem cells improved clinical manifestations in progressive MS patients. It was found that six of these eight patients showed therapeutic improvement after hUC-MSC transplantation, as evaluated by EDSS, and relapses in all eight patients were reduced significantly, indicating that HU-MSCs could be a potential therapeutic option for patients with progressive MS. 


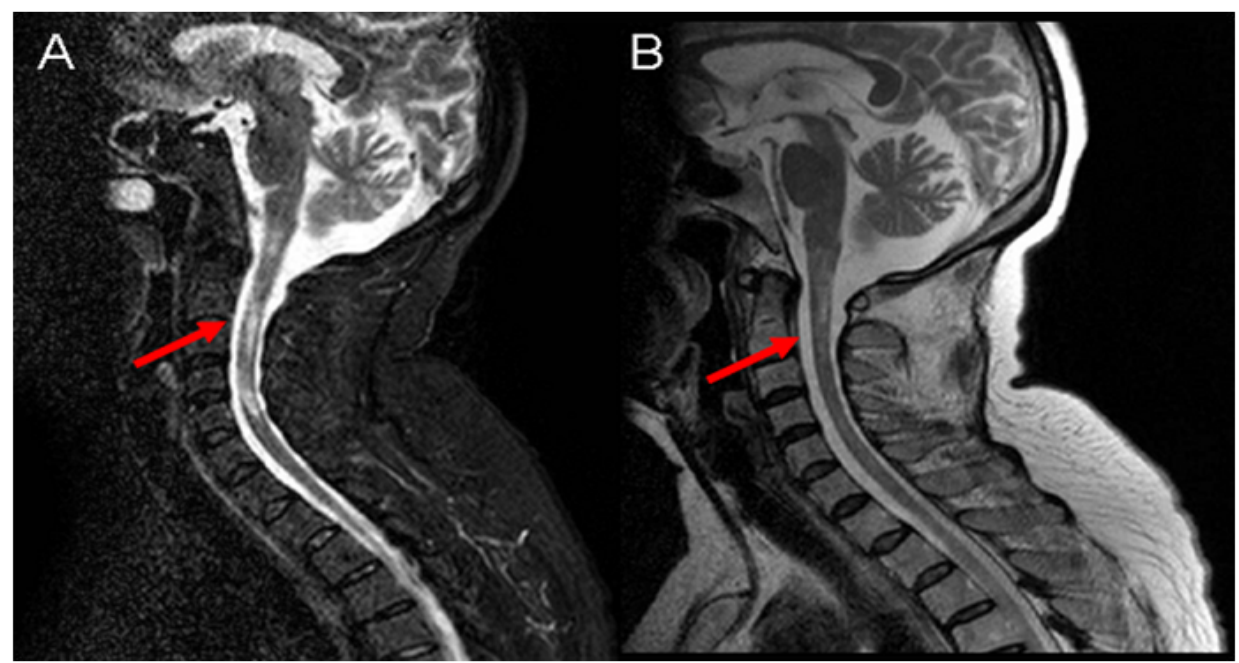

Figure 5: Neuroradiological effects. (A) T2-weighted MRI showed diffuse and multifocal intramedullary lesions in the brain stem and spinal cord before transplantation (red arrow). (B) T2-weighted MRI 12 months after transplantation: the scope and number of lesions significantly decreased.

Currently, various treatments have emerged with the hope of alleviating and reversing progression of MS. Until now, interferon's such as interferon beta- $1 \mathrm{~b}$ and interferon beta-1a are approved for treatment of SPMS in Europe, whereas they are not in the USA. Moreover, mitoxantrone was approved and can also be used for SPMS, but studies proved these treatments were effective in the early progressive phase with ongoing relapses. For later stages of progressive phase, therapies are rare or not available $[1,15]$. In this current study, all patients were treated with high-dose methylprednisolone (MP), and other therapies including intravenous immunoglobulin, interferons, azathioprine and cyclophosphamide (CYC), but the disease duration is still converted into a progressive process.

Against this background, human stem cells have attracted more attention. Research showed that stem cell therapy had huge potential for immunomodulatory, trophic factor secretory properties, boosting remyelination of multiple sclerosis and, finally, regenerating damaged nervous tissue and reversing accumulated disability [16].

Mesenchymal stem cells (MSCs) have long been considered the main stem cell sources in clinical trials. Accumulating clinical studies have shown that MSCs are beneficial in MS [17]. Autologous BM-MSCs in 10 patients with advanced MS indicated clinical improvement, but MRIs revealed new or enlarging lesions during 3-6 months [18]. Autologous stem cell transplantation stabilized or improved progressive MS in 63\% of patients [19]. These reports also demonstrated that MSCs had no serious adverse effects as evidenced by no detectable tumor formation.

Human umbilical cord mesenchymal stem cells (hUC-MSCs), isolated from human umbilical cords, are earlier-stage cells than MSCs derived from adult fat or bone marrow and have the potential of neuroprotection and immunomodulation. Compared to human bone marrow mesenchymal stem cells (BM-MSC), the number of potential donors of hUC-MSCs is higher; the collection of hUC-MSCs is more convenient and non-invasive. Moreover, hUC-MSCs self-renew faster, are more primitive than other MSCs and the lower immunogenicity of the cells reduced graft-versus-host reactivity. Furthermore, use of hUC-MSCs raises no ethical issues [20,21].

This study demonstrates that hUC-MSCs could be used to alleviate symptoms and reduce relapses in patients with progressive MS.
Meanwhile, MRI scans revealed that lesions, compared with baseline, became smaller twelve months after transplantation. Gadoliniumenhanced lesions, detected in MRIs and proved to be associated with intense inflammatory activity and dense perivascular cuffs, can therefore be used as a useful surrogate measure of relapses and an outcome criterion in many therapeutic trials of MS [22]. In this study, the Gd enhancing lesions were decreased in two patients, suggesting the efficacy of hUC-MSCs.

Although the direct roles and mechanism of the hUC-MSCs in affording therapeutic effects in response to autoimmune injury and demyelination are not well-defined, the result of lymphocyte classification in this study showed that B cells and T cells of blood were inhibited after hUC-MSCs treatment in all six improved patients. These findings indicated that the effect of hUC-MSCs might be related with immunomodulatory.

Inflammation is the driving force for CNS injury in patients with SPMS and research found a highly significant correlation between inflammation (T-cells, B-cells, or macrophages) and neurodegeneration [23]. CNS intrinsic innate inflammation is responsible for progression, appears to drive tissue degeneration and causes disability in progressive MS [24]. Some researchers pointed out that inability to cross the bloodbrain barrier and target the inflammatory cells was the reason why some treatments failed to treat RRMS [25]. The possible mechanism of hUC-MSCs exerting immune modulatory effects in patients with SPMS may be that hUC-MSCs can cross the blood-brain barrier via intravenous transplantation, down regulated LINGO-1 mRNA and protein expression to regulate CNS axon growth and facilitate remyelination $[25,26]$. The precise mechanism still needs further study.

The current study evaluated the safety profile of hUC-MSC transplantation for the eight patients who received the treatment for 18 months and it was found that few adverse reactions occurred. Generally, HBMSCs are considered to enhance tumorigenesis since they are a source of carcinoma-associated fibroblasts. No tumor generation or virus inflammation was found after 18 month followup, which was consistent with the clinical trial involving HUC-MSCs in patients with severe refractory systemic lupus erythematosus [27]. Further, there is no immune-rejection for this therapy, suggesting that hUC-MSCs could be used as allogenic cells. 
Citation: Lu Z, Zhao H, Xu J, Zhang Z, Zhang X, et al. (2013) Human Umbilical Cord Mesenchymal Stem Cells in the Treatment of Secondary Progressive Multiple Sclerosis. J Stem Cell Res Ther S6: 002. doi:10.4172/2157-7633.S6-002

In summary, this study proved that hUC-MSC transplantation is effective on progressive MS in the short term and may be safe. However, only eight patients were enrolled in this study. A larger clinical trial with more patients at multicenter and longer follow-up would be required to further assess these encouraging preliminary results.

\section{Conflict of Interest}

There are no conflicts of interest for any of the authors relating to this manuscript.

\section{Acknowledgment}

This study was supported by the National Natural Science Foundation of China $(81171085,30971010$ and 81100863$)$, the Medical Leading Talent and Innovation Team Project of Jiangsu Province (LJ201101), the Natural Science Foundation (BK2009037) of Jiangsu Province of China, Science and Technology Achievement Transformation Foundation of Jiangsu Province (BA2009124) and Medical Technology Development Project of Nanjing (ZKX09022, ZKX08030).

\section{References}

1. Rommer PS, Stüve O (2013) Management of Secondary Progressive Multiple Sclerosis: Prophylactic Treatment-Past, Present, and Future Aspects. Curr Treat Options Neurol 15: 241-258.

2. Murray TJ (2009) The history of multiple sclerosis: the changing frame of the disease over the centuries. J Neurol Sci 277: S3-8.

3. Vermersch P, Benrabah R, Schmidt N, Zéphir H, Clavelou P, et al. (2012) Masitinib treatment in patients with progressive multiple sclerosis: a randomized pilot study. BMC Neurol 12: 36.

4. Bradl M, Lassmann H (2009) Progressive multiple sclerosis. Semin Immunopathol 31: 455-465.

5. Sng J, Lufkin T (2012) Emerging Stem Cell Therapies: Treatment, Safety, and Biology. Stem Cells Int 2012: 521343.

6. Auletta JJ, Bartholomew AM, Maziarz RT, Deans RJ, Miller RH, et al. (2012) The potential of mesenchymal stromal cells as a novel cellular therapy for multiple sclerosis. Immunotherapy 4: 529-547.

7. Hou Y, Ryu CH, Park KY, Kim SM, Jeong CH, et al. (2013) Effective combination of human bone marrow mesenchymal stem cells and minocycline in experimental autoimmune encephalomyelitis mice. Stem Cell Res Ther 4: 77.

8. Friedman R, Betancur M, Boissel L, Tuncer H, Cetrulo C, et al. (2007) Umbilical Cord Mesenchymal Stem Cells: Adjuvants for Human Cell Transplantation. Biol Blood Marrow Transplant 13: 1477-1486.

9. Chao KC, Yang HT, Chen MW (2012) Human umbilical cord mesenchymal stem cells suppress breast cancer tumorigenesis through direct cell-cell contact and internalization. J Cell Mol Med 16: 1803-1815.

10. McDonald WI, Compston A, Edan G, Goodkin D, Hartung HP, et al. (2001) Recommended diagnostic criteria for multiple sclerosis: guidelines from the International Panel on the diagnosis of multiple sclerosis. Ann Neurol 50: 121127.

11. Polman CH, Reingold SC, Edan G, Filippi M, Hartung HP, et al. (2005) Diagnostic criteria for multiple sclerosis: 2005 revisions to the "McDonald Criteria". Ann Neurol 58: 840-846.
12. Kurtzke JF (1983) Rating neurologic impairment in multiple sclerosis: an expanded disability status scale (EDSS). Neurology 33: 1444-1452.

13. Barkhof F, Filippi M, Miller DH, Scheltens P, Campi A, et al. (1997) Comparison of MRI criteria at first presentation to predict conversion to clinically definite multiple sclerosis. Brain 120: 2059-2069.

14. Polman CH, Reingold SC, Banwell B, Clanet M, Cohen JA, et al. (2011) Diagnostic criteria for multiple sclerosis: 2010 revisions to the "McDonald Criteria". Ann Neurol 69: 292-302.

15. Kantarci O (2013) Treatment of Primary Progressive Multiple Sclerosis. Semin Neurol 33: 74-78.

16. Broadley SA (2013) Multiple Sclerosis: From Molecules to Treatment. Int J Mo Sci 14: 7598-7602.

17. Karussis D, Karageorgiou C, Vaknin-Dembinsky A, Gowda-Kurkalli B, Gomor JM, et al. (2010) Safety and immunological effects of mesenchymal stem cell transplantation in patients with multiple sclerosis and amyotrophic lateral sclerosis. Arch Neurol 67: 1187-1194.

18. Yamout B, Hourani R, Salti H, Barada W, El-Hajj T, et al. (2010) Bone marrow mesenchymal stem cell transplantation in patients with multiple sclerosis: a pilot study. J Neuroimmunol 227: 185-189.

19. Saccardi R, Kozak T, Bocelli-Tyndall C, Fassas A, Kazis A, et al. (2006) Autologous stem cell transplantation for progressive multiple sclerosis: update of the European Group for Blood and Marrow Transplantation autoimmune diseases working party database. Mult Scler 12: 814-823.

20. Malgieri A, Kantzari E, Patrizi MP, Gambardella S (2010) Bone marrow and umbilical cord blood human mesenchymal stem cells: state of the art. Int J Clin Exp Med 3: 248-69.

21. Kim J, Shin JM, Jeon YJ, Chung HM, Chae JI (2012) Proteomic Validation of Multifunctional Molecules in Mesenchymal Stem Cells Derived from Human Bone Marrow, Umbilical Cord Blood and Peripheral Blood. PLoS One 7: e32350.

22. Fitzner D, Simons M (2010) Chronic Progressive Multiple SclerosisPathogenesis of Neurodegeneration and Therapeutic Strategies. Curr Neuropharmacol 8: 305-315.

23. Frischer JM, Bramow S, Dal-Bianco A, Lucchinetti CF, Rauschka H, et al. (2009) The relation between inflammation and neurodegeneration in multiple sclerosis. Brain 132: 1175-1189.

24. Lassmann $\mathrm{H}$ (2012) Cortrical lesions in multiple sclerosis: inflammation vs neurodegeneration. Brain 135: 2904-2905.

25. Zhang MJ, Sun JJ, Qian L, Liu Z, Zhang Z, et al. (2011) Human umbilical mesenchymal stem cells enhance the expression of neurotrophic factors and protect ataxic mice. Brain Res 1402: 122-131.

26. Liu R, Zhang Z, Lu Z, Borlongan C, Pan J, et al. (2013) Human Umbilica Cord Stem Cells Ameliorate Experimental Autoimmune. Encephalomyelitis by Regulating Immunoinflammation and Remyelination. Stem Cells Dev 22: 1053-1062.

27. Sun L, Wang D, Liang J, Zhang H, Feng X, et al. (2010) Umbilical cord mesenchymal stem cell transplantation in severe and refractory systemic lupus erythema tosus. Arthritis Rheum 62: 2467-2675.
This article was originally published in a special issue, Transplantation Immunology handled by Editor(s). Qing Ma, University of Texas, USA 